\title{
Singlet Fission Dynamics Modulated by Molecular Configuration in Covalently Linked Pyrene Dimers, Anti- and Syn-1,2-di(Pyrenyl)Benzene
}

\section{Jungkweon Choi}

Institute for Basic Science

Siin Kim

Institute for Basic Science

Mina Ahn

Daegu University

Jungmin Kim

Institute for Basic Science

Dae Won Cho

Korea University

\section{Kyung-Ryang Wee}

Institute for Basic Science

Hyotcherl lhee ( $\nabla$ hyotcherl.ihee@kaist.ac.kr)

Korea Advanced Institute of Science and Technology (KAIST) https://orcid.org/0000-0003-0397-5965

\section{Article}

Keywords: Singlet Fission, Molecular Configuration, Pyrene Dimers, (pyrenyl)benzene, structural isomers, potential material, real devices, power conversion

Posted Date: October 28th, 2021

DOI: https://doi.org/10.21203/rs.3.rs-988842/v1

License: (c) (1) This work is licensed under a Creative Commons Attribution 4.0 International License. Read Full License 


\section{Singlet Fission Dynamics Modulated by Molecular Configuration in Covalently Linked Pyrene Dimers, Anti- and Syn-1,2-di(pyrenyl)benzene}

Jungkweon Choi, ${ }^{a, b}$ Siin Kim, ${ }^{a, b}$ Mina Ahn, ${ }^{c}$ Jungmin Kim, ${ }^{a, b}$ Dae Won Cho, ${ }^{d}$ Kyung-Ryang Wee, * ${ }^{c}$ Hyotcherl Ihee ${ }^{* a, b}$

a Center for Nanomaterials and Chemical Reactions, Institute for Basic Science, Daejeon 34141, Republic of Korea

b Department of Chemistry and KI for the BioCentury, Korea Advanced Institute of Science and Technology (KAIST), Daejeon 305-701, Republic of Korea

c Department of Chemistry and Institute of Natural Science, Daegu University, Gyeongsan 38453, Republic of Korea

d Department of Advanced Materials Chemistry, Korea University, Sejong Campus, Sejong 30019, Korea

* Corresponding authors: Kyung-Ryang Wee and Hyotcherl Ihee

E-mail: krwee@daegu.ac.kr, hyotcherl.ihee@kaist.ac.kr 


\begin{abstract}
Various covalently linked dimers (CLDs) and their structural isomers have attracted much attention as a potential material to improve the power conversion efficiencies of photovoltaic devices and OLEDs through singlet fission (SF). However, the effect of the molecular configuration on improving the efficiency through the spatial arrangement has not been explored yet. Here, we designed and synthesized two covalently ortho-linked pyrene (Py) dimers, anti- and syn-1,2-di(pyrenyl)benzene (Anti-DPyB and Syn-DPyB), to elucidate the effect of the molecular configuration on SF dynamics. Both Anti-DPyB and Syn-DPyB, which have different Py-stacking configurations, form excimers and then relax to the correlated triplet pair $\left({ }^{1}\left(\mathrm{~T}_{1} \mathrm{~T}_{1}\right)\right)$ state, indicating the occurrence of SF. Unlike previous studies where the excimer formation inhibited an SF process, the ${ }^{1}\left(\mathrm{~T}_{1} \mathrm{~T}_{1}\right)$ 's of Anti-DPyB and Syn-DPyB are formed through the excimer state. The ${ }^{1}\left(\mathrm{~T}_{1} \mathrm{~T}_{1}\right)$ of Anti-DPyB dissociates to form free triplets, completing SF, whereas the ${ }^{1}\left(\mathrm{~T}_{1} \mathrm{~T}_{1}\right)$ of Syn-DPyB does not undergo dissociation. Our results showcase that the molecular configuration of a CLD plays an important role in SF dynamics, suggesting that the strategic molecular design and its experimental characterization may provide a new route to improving the power conversion efficiency in real devices.
\end{abstract}




\section{Introduction}

The electron (or charge) carrier dynamics in photoelectric and electrochemical devices are key to determining the performance of devices. ${ }^{1,2,3,4,5,6}$ Chromophore-chromophore interaction as well as the electronic-state coupling of a chromophore can modulate such electron carrier dynamics. ${ }^{7}$, 8, 9, 10,11 In this regard, many multi-chromophore systems have been widely used for developing highly efficient photoelectric or electrochemical devices using chromophore-chromophore interaction. ${ }^{7}$ 12, 13, 14, 15, 16 Among multi-chromophore systems, covalently linked dimers (CLDs) have attracted much attention as potential materials to provide high energy conversion efficiencies in photovoltaic devices because their excited-state relaxation dynamics, such as the excimer formation, intramolecular charge transfer (ICT), and singlet fission (SF), can be modulated by the strategic molecular design. ${ }^{9}, 17,18,19,20$ Especially the dynamics of SF, which is a conversion process from one singlet exciton into two triplet excitons, have been actively investigated with various time-resolved spectroscopies, to overcome the limit of Shockley-Queisser power conversion efficiency. ${ }^{12,21,22,23,24,25}$

Recent studies reported that SF takes place through various types of species such as a chargetransfer species, an excimer, and higher excited vibrational and electronic states. ${ }^{16,19,20,22,24,26,27,}$ 28, 29 Zirzlmeier et al. suggested that the SF process occurring in ortho-, meta-, and para-linked pentacene dimers proceeds through virtual CT states. ${ }^{22}$ Margulies et al. reported that the covalently linked terrylene-3,4:11,12-bis(dicarboximide) (TDI) dimer with a stacked structure forms an excimer within $<200 \mathrm{fs}$, whereas the slip-stacked TDI dimer in a nonpolar solvent forms the correlated triplet pair $\left({ }^{1}\left(\mathrm{~T}_{1} \mathrm{~T}_{1}\right)\right)$, which is an intermediate in the SF process. ${ }^{23}$ They also showed that the slip-stacked TDI dimer forms a CT state in a few picoseconds in a polar solvent, suggesting that adjusting the CT state energy relative to exciton states via solute-solvent interaction can either promote or inhibit SF. ${ }^{23}$ The experimental and theoretical calculation results on various CLDs show that compared with ortho- and para-linked dimers, the meta-linked dimers exhibit more efficient SF dynamics due to the small binding energy $\left(E_{\mathrm{b}}\right)$ of $\left.{ }^{1}\left(\mathrm{~T}_{1} \mathrm{~T}_{1}\right)\left(E_{\mathrm{b}}=2 E\left|\mathrm{~S}_{0} \mathrm{~T}_{1}\right\rangle-\left.E\right|^{1} \mathrm{~T}_{1} \mathrm{~T}_{1}\right\rangle\right){ }^{22}$, 30,31,32 Contrary to the theoretical prediction and experimental results for CLDs, Korovina et al. showed that the ortho- and para-bis(ethynyltetracenyl)benzene dimers exhibit SF, while the metabis(ethynyltetracenyl)benzene dimer predominantly undergoes a radiative decay. Especially, unlike ortho-linked dimer, which forms only the ${ }^{1}\left(\mathrm{~T}_{1} \mathrm{~T}_{1}\right)$, the para-linked dimer shows the 
complete SF dynamics to form free triplets. ${ }^{33} \mathrm{Ni}$ et al. reported that upon excitation at $250 \mathrm{~nm}$ the cofacial perylene dimer undergoes SF from the upper excited vibrational and electronic states, whereas upon excitation at $450 \mathrm{~nm}$ it fast forms an excimer, which relaxes to the ground state in nanoseconds time scale. ${ }^{34}$

As can be seen in these examples, the results from numerous experimental and theoretical approaches for CLDs show diverse and inconsistent SF dynamics. Accordingly, in-depth studies are needed to understand their excited-state relaxation dynamics, including SF. Besides, the study on the effect of a molecular configuration, which can affect its excited-state relaxation dynamics, may provide a clue for the optimized spatial arrangements for the high energy conversion efficiency of a real device, but such studies are rare. In this regard, the effect of a molecular configuration on the excited-state relaxation dynamics of a CLD should be studied first of all. With this perspective, we designed and synthesized two covalently ortho-linked pyrene (Py) dimers, anti- and syn-1, 2-di(pyrenyl)benzene (Anti-DPyB and Syn-DPyB) (see Supplementary Information and Figure 1 and Supplementary Figures S1-S6), which were expected to have different configurations, to elucidate the effect of the molecular configuration for their excitedstate relaxation dynamics using various steady-state and time-resolved spectroscopies. The data show that both Anti-DPyB and Syn-DPyB form excimers, which rapidly relax to the correlated triplet pair $\left({ }^{1}\left(\mathrm{~T}_{1} \mathrm{~T}_{1}\right)\right)$ state regardless of the solvent polarity, indicating the occurrence of $\mathrm{SF}$ dynamics. Notably, ${ }^{1}\left(\mathrm{~T}_{1} \mathrm{~T}_{1}\right)$ 's of Anti-DPyB in both $n$-hexane and acetonitrile are dissociated to free triplets as the end product, completing SF, whereas Syn-DPyB does not show the dissociation of ${ }^{1}\left(\mathrm{~T}_{1} \mathrm{~T}_{1}\right)$ in both $n$-hexane and acetonitrile, indicating that the ${ }^{1}\left(\mathrm{~T}_{1} \mathrm{~T}_{1}\right)$ in $S y n$-DPyB is bound with respect to the separated triplets. This study showcases that the different SF dynamics of Anti-and Syn-DPyB are due to the different molecular configurations of Anti-DPyB and Syn-DPyB.

\section{Results}

\section{Molecular structures.}

To characterize structures of $A n t i-\mathrm{DPyB}$ and $S y n-\mathrm{DPyB}$, we calculated their minimum energy structures using density functional theory (DFT). The optimized structures are shown in Figure 1. Two Py moieties in Anti-DPyB are far away from each other, whereas Syn-DPyB shows a partial overlap of two pre-stacked Py moieties. According to the calculations using B3LYP/6-31G(d,p), the distances $\left(r_{\mathrm{C} 1-\mathrm{C} 2}\right)$ between two Py moieties in 
Anti-DPyB and Syn-DPyB are 10.326 and $8.481 \AA$, respectively. This result is consistent with the calculation results reported by Jo et al. ${ }^{35}$ While the calculation results for AntiDPyB and Syn-DPyB depend on the calculation method, they show the common feature that the $r_{\mathrm{C} 1-\mathrm{C} 2}$ of Anti-DPyB is longer than that of Syn-DPyB. The optimized structure of Anti-DPyB obtained from our calculation is similar to its crystal structure as well (Supplementary Figure S7).

\section{Steady-state absorption/emission spectra.}

To elucidate the photophysical properties of Anti-DPyB and Syn-DPyB, we measured their UV-visible absorption spectra in $n$-hexane and acetonitrile. As shown in Figure 1B, AntiDPyB shows two vibrationally resolved absorption bands at around $250-280$ and 300 $380 \mathrm{~nm}$. This feature is similar to the absorption spectrum of 1-phenylpyrene (Ph-Py). ${ }^{9,} 36$ In contrast, Syn-DPyB exhibits a broad and vibrationally resolved absorption band at 250 $475 \mathrm{~nm}$. The vibrationally resolved absorption bands observed from Anti-DPyB and SynDPyB indicate that both compounds have highly rigid structures in the ground state. Furthermore, the absorption bands of Anti-DPyB and Syn-DPyB do not show noticeable dependence on solvent polarity (Figure 1B), indicating that the interaction between the solute and the solvent molecules in the ground state is not strong enough to affect the photophysical property of the solute molecule.

To investigate the excited-state behaviors of Anti-DPyB and Syn-DPyB, their emission spectra in $n$-hexane and acetonitrile were measured with excitation at $345 \mathrm{~nm}$, which corresponds to the major absorption peak. Anti-DPyB and Syn-DPyB in both solvents show dual emission bands ( $\sim 380 \mathrm{~nm}$ and $\sim 480 \mathrm{~nm}$ for Anti-DPyB and $\sim 420 \mathrm{~nm}$ and $\sim 480 \mathrm{~nm}$ for Syn-DPyB), detailed features of which are not the same. The $\sim 380 \mathrm{~nm}$ band of Anti-DPyB is structured whereas the broad band centered at $\sim 480 \mathrm{~nm}$ is structureless (Figure 1B). Unlike Anti-DPyB, both of the two emission bands of Syn-DPyB are structured. Based on numerous previous studies on Py and Py derivatives, ${ }^{37,38}$ the shorter-wavelength ( $\sim 380 \mathrm{~nm}$ and $\sim 420 \mathrm{~nm}$ ) emissions from Anti-DPyB and Syn-DPyB can be assigned to Py monomer moieties and the longer-wavelength ( $\sim 80 \mathrm{~nm}$ for both) emissions to the excimer formed via the association of the excited Py and the unexcited Py. In addition, the broad structureless bands centered at $\sim 480 \mathrm{~nm}$ observed from Anti-DPyB are highly similar to the 
typical excimer bands observed from Py and Py derivatives. In terms of solvent dependence, in Anti-DPyB, the relative intensities of the emissions from the monomeric Py moiety and the excimer show significant dependence on solvent polarity, whereas Syn-DPyB does not show definite solvent dependency.

\section{Fluorescence lifetime.}

To further elucidate the excited-state relaxation dynamics, the fluorescence decay profiles of $A n t i$-DPyB and Syn-DPyB in $n$-hexane and acetonitrile were measured. As depicted in Figure 2, all decay profiles were satisfactorily fit with bi-exponential functions. The determined fluorescence lifetimes are summarized in Table 1. The fast $\left(\tau_{\mathrm{f} 1}\right)$ and slow $\left(\tau_{\mathrm{f} 2}\right)$ time constants are dominantly observed in the shorter- and longer-wavelength emissions, respectively. Since the shorter- and longer-wavelength emissions arise from the Py monomeric unit and excimer, respectively, the fast $\left(\tau_{\mathrm{f} 1}\right)$ and slow $\left(\tau_{\mathrm{f} 2}\right)$ time constants correspond to the fluorescence lifetimes of the Py monomeric unit and excimer, respectively. In addition, as depicted in Figure 2A, the fluorescence decay profile of AntiDPyB measured at the long-wavelength region $(500 \sim 600 \mathrm{~nm})$ in $n$-hexane shows an additional kinetic component with a rise time of $1.24 \mathrm{~ns}$.

\section{Time-resolved TA spectrum.}

To elucidate the excited state relaxation dynamics, we measured the femtosecond transient absorption (fs-TA) spectra for Anti-DPyB and Syn-DPyB in $n$-hexane and acetonitrile with $350 \mathrm{~nm}$ excitation. As shown in Figure 3 and Supplementary Figure S8, the TA spectra of Anti-DPyB in $n$-hexane and acetonitrile exhibit broad signals at $400-700 \mathrm{~nm}$ corresponding to the excited state absorption (ESA). With time, these broad positive signals transform into structured signals. On the other hand, the TA spectra of Syn-DPyB in $n$ hexane and acetonitrile exhibit intense ESA signals at $450-530 \mathrm{~nm}$ with a weak absorption tail $(550-700 \mathrm{~nm})$. To extract further information, we analyzed the TA spectra of AntiDPyB and Syn-DPyB using singular value decomposition (SVD) analysis (see the Supplementary Information). The SVD analysis for TA spectra of Anti-DPyB and SynDPyB identifies four and three significant singular components, respectively (see Supplementary Figures S9 - S10). As shown in Supplementary Figure S11, the significant 
rSVs for Anti-DPyB in $n$-hexane and acetonitrile can be expressed by a tetra-exponential function with shared relaxation times $(3.6 \pm 0.3 \mathrm{ps}, 231 \pm 19 \mathrm{ps}, 1.75 \pm 0.12 \mathrm{~ns}$, and $>10 \mathrm{~ns}$ in $n$-hexane; $2.8 \pm 0.1 \mathrm{ps}, 24.3 \pm 0.5 \mathrm{ps}, 495.7 \pm 6.5 \mathrm{ps}$, and $>10 \mathrm{~ns}$ in acetonitrile). The significant rSVs for Syn-DPyB in $n$-hexane and acetonitrile can be expressed by a triexponential function with shared relaxation times $(2.3 \pm 0.8 \mathrm{ps}, 9.7 \pm 0.5 \mathrm{ps}$, and $6.4 \pm 0.2$ ns in n-hexane; $2.8 \mathrm{ps}, 8.0 \pm 0.6 \mathrm{ps}$, and $4.8 \pm 0.2 \mathrm{~ns}$ in acetonitrile) (see Supplementary Figure S11). Time constants are summarized in Table 2. Details regarding SVD and kinetic analysis, including a discussion of the kinetic model, are given in the experimental section of the Supplementary Information, SVD analysis results (Supplementary Figures S9 - S10) and fits of rSVs (Supplementary Figure S11) are given in the Supplementary Information.

To observe the long-lived triplet excited states, furthermore, we measured nanosecond TA spectra of Anti-DPyB and Syn-DPyB in $n$-hexane and acetonitrile with $355 \mathrm{~nm}$ excitation. Anti-DPyB in $n$-hexane and acetonitrile show a weak and broad absorption band around $445 \mathrm{~nm}$ at a few microseconds time delays (Supplementary Figure S12), suggesting the presence of a long-lived species such as a triplet species. In contrast, Syn-DPyB does not exhibit any absorption band in both $n$ - hexane and acetonitrile, indicating there is no long-lived species such as a triplet species at the $\mu$ s - ms time scales.

\section{Discussion}

\section{Intramolecular excimer formation.}

The optimized structures of Anti-DPyB and Syn-DPyB show that the $r_{\mathrm{C} 1-\mathrm{C} 2}$ in Anti-DPyB is longer than that in Syn-DPyB, suggesting Anti-DPyB should have the weaker interaction between two Py moieties than Syn-DPyB with the partial overlap of two Py moieties. Accordingly, Anti-DPyB is likely to show similar photophysical properties to Py or 1phenyl pyrene (Ph-Py). ${ }^{9}{ }^{36}$ Indeed, Anti-DPyB shows the absorption spectrum similar to those of Py or Ph-Py, indicating that in the ground state, Anti-DPyB has a monomeric character. In contrast, the pre-stacked structure of Syn-DPyB likely induces significantly different photophysical properties compared with $\mathrm{Py}$ or $\mathrm{Ph}-\mathrm{Py}$. Compared to $\mathrm{Py}, \mathrm{Ph}-\mathrm{Py}$, or Anti-DPyB, Syn-DPyB exhibits a single broad and vibrationally resolved absorption band at $250-475 \mathrm{~nm}$ owing to the strong $\pi-\pi$ interaction between two Py moieties. As a result, the excited state of Syn-DPyB is expected to show the characteristic feature of an excimer. 
These steady-state spectroscopic results confirm this prediction that in the ground state, the interaction between two Py moieties in Anti-DPyB is much weaker than that in Syn-DPyB. The difference in the interaction between two Py moieties in Anti-DPyB and Syn-DPyB greatly influences the formation of an excimer in terms of the structural rigidity of the excimer and the solvent dependence of the emission spectra, as shown in various experimental data discussed below.

We note that the excimer formation in Anti-DPyB and Syn-DPyB is intramolecular. Therefore, it is predicted that the emission spectra should not show strong dependence on the concentration, unlike the emission spectra of the excimer formed by the association of monomers. Since the excimer formation is a short-range interaction, it requires molecular contact between chromophores. Consequently, for monomer solutions, the excimer is easily formed in a high concentration solution rather than in a low concentration solution. On the contrary, the emission spectra of Anti-DPyB and Syn-DPyB are not influenced by the solute concentration (Supplementary Figure S13). This result confirms that the excimer formation in Anti-DPyB and Syn-DPyB is due to the intramolecular interaction rather than the intermolecular interaction. Since in Anti-DPyB, the interaction between two Py moieties in the ground state is weak due to the long distance between two chromophores with twisted alignment, the intramolecular excimer formation requires the rearrangement of two distant Py moieties. This scenario is consistent with the flexible structure of the excimer of AntiDPyB reflected on its broad structureless excimer emission band ( 480 nm). In contrast, we expect that Syn-DPyB rapidly forms the excimer with no or less structural rearrangement because of the partial overlap of two Py moieties. Based on the structured excimer emission band ( $480 \mathrm{~nm})$ of $S y n-\mathrm{DPyB}$, we suggest that the excimer structure is as rigid as the structure in the ground state.

The solvent dependence on the emission spectra of Anti-DPyB can be rationalized by considering the following scenario. As Py is a hydrophobic molecule, two Py moieties in a nonpolar solvent show a monomeric behavior, whereas a high polarity solvent facilitates the interaction of two Py moieties, resulting in the more efficient excimer formation. The absence of solvent dependence on the emission of $S y n$-DPyB is probably due to its rigid structure owing to the strong $\pi-\pi$ interaction between two Py moieties. Unlike Anti-DPyB and Syn-DPyB that form the excimer in the excited state, 1,4-di(1-pyrenyl)benzene (Py- 
Benz-Py), which is a covalently para-linked pyrene (Py) dimer, shows significantly different excited-state relaxation dynamics. ${ }^{9}$ Due to the structure of Py-Benz-Py, where the two Py moieties are far apart without any overlap, Py-Benz-Py is not expected to form the intramolecular excimer formation. Indeed, it was reported that Py-Benz-Py exhibits the solvent-dependent ICT dynamics, followed by the twisting motion between Py and phenyl moieties, without the intramolecular excimer formation. ${ }^{9}$ The difference in the excited-state relaxation dynamics of Anti-DPyB, Syn-DPyB and Py-Benz-Py indicates that the molecular structure and configuration play a vital role in their excited-state relaxation dynamics.

\section{Excited-state dynamics dependent on molecular configuration.}

As shown in Table 2, the TA measurements for both Anti-DPyB and Syn-DPyB show similar $\tau_{1}$ time constants $(2.3 \sim 3.7 \mathrm{ps})$ regardless of solvent polarity. This time scale falls into the well-known time scale for vibrational relaxation. Thus, the earliest kinetic component $\left(\tau_{1}\right)$ of $\sim 3$ ps can be interpreted as the intramolecular vibrational relaxation (IVR) from the initially populated local excited state (Franck-Condon state).

After IVR, the excited molecules in the $S_{1}$ state have various potential fates, including relaxation to other excited states, such as excimer or triplet excited states, and returning to the ground state via fluorescence $\left(S_{1} \rightarrow S_{0}\right)$. The observation of the excimer fluorescence for Anti-DPyB and Syn-DPyB leads to the interpretation that a part of the excited molecules in the $\mathrm{S}_{1}$ state relaxes to the excimer state. As discussed in the section of Intramolecular excimer formation, the excimer formation in Anti-DPyB should require rearranging two distant Py moieties to induce the interaction between two distant Py moieties. Such

rearrangement to form the excimer is likely to be the twisting motion between Py and phenyl moiety. In this regard, the $\tau_{2}$ time constants $(24.3 \sim 231 \mathrm{ps})$ observed from Anti$\mathrm{DPyB}$ can be interpreted in terms of the conformational change. We note that the $\tau_{2}$ time constant (231 ps) of Anti-DPyB in $n$-hexane is similar to $323 \mathrm{ps}$ assigned to the twisting motion between Py and phenyl moiety of Py-Benz-Py in a nonpolar solvent. Thus, we attribute $\tau_{2}$ of Anti-DPyB to the excimer formation via the $S_{1} \rightarrow$ excimer transition accompanying the twisting motion between Py and phenyl moiety. The faster excimer formation in acetonitrile is probably due to the strong hydrophobic interaction between two Py moieties in a high-polarity solvent. 
The second fastest time constant ( 9.7 and 8.0 ps in $n$-hexane and acetonitrile, respectively) for Syn-DPyB is faster than those for Anti-DPyB by two orders of magnitude. Nevertheless, these time scales are much longer than the typical sub-picosecond time scale reported for the excimer formation of pre-stacked dimeric systems. ${ }^{19,}{ }^{23}$ Several studies reported ultrafast excimer formation in CLDs. ${ }^{19,23}$ For example, Hong et al. showed that the excimer state of cofacial stacked perylene bisimide dimer is formed within 200 fs. ${ }^{19}$ Unlike Anti-DPyB, the excimer in Syn-DPyB with a pre-stacked structure should be rapidly formed with no or less structural rearrangement, and thus we surmise two possibilities: the excited molecules in $\mathrm{S}_{1}$ formed by IVR ( $\left.3 \mathrm{ps}\right)$ relax to the excimer state (i) within a subpicosecond ( $\leq 200 \mathrm{fs})$, and (ii) with a time constant comparable to IVR ( $\sim 3 \mathrm{ps})$. For this reason, we ruled out the possibility that the second fastest time constants in Syn-DPyB are attributed to the excimer formation dynamics. Notably, the TA spectra of a long-lived species formed with these time constants in Syn-DPyB and those of a long-lived species formed with $\tau_{3}$ time constants in Anti-DPyB are highly similar to the triplet-triplet absorption spectra of free Py derivatives corresponding to the $\mathrm{T}_{1} \rightarrow \mathrm{T}_{\mathrm{n}}$ transition. ${ }^{39,40}$ For this reason, we denote time constants of 9.7 and 8.0 ps for $S y n$-DPyB as $\tau_{3}$, not $\tau_{2}$. In other words, the long-lived species in Anti-DPyB and Syn-DPyB are formed with $\tau_{3}$ time constants. The long-lived species of Anti-DPyB and Syn-DPyB show structured TA spectra, similar to the $\mathrm{T}_{1}$-to- $\mathrm{T}_{\mathrm{n}}$ absorption spectra for carbonylpyrenes reported by Rajagopal et al. ${ }^{40}$ Furthermore, as shown in Supplementary Figure S14, the TA signals of Anti-DPyB observed at $>5 \mathrm{~ns}$ resemble the $\mathrm{T}_{1}$-to- $\mathrm{T}_{\mathrm{n}}$ absorption spectrum of 1-(2-bromophenyl)pyrene measured in dichloromethane (DCM), although the peak positions are slightly different from each other. Therefore, the long-lived species observed in Anti-DPyB and Syn-DPyB are $\mathrm{T}_{1}$ (free triplet state) or a similar state that gives an absorption spectra similar to that of $\mathrm{T}_{1}$-to- $\mathrm{T}_{\mathrm{n}}$.

Free triplet states of a molecule can be generated through an ISC process or the dissociation of ${ }^{1}\left(\mathrm{~T}_{1} \mathrm{~T}_{1}\right)$, which is an intermediate formed in the SF process. Generally, it has been accepted that ISC in organic molecules with a small spin-orbit coupling (SOC) occurs with a timescale of $10 \mathrm{~ns}$ to $1 \mathrm{~ms}$ and that the lifetime of the triplet state is longer than the order of $1 \mu \mathrm{s}$. In contrast to ISC, the ${ }^{1}\left(\mathrm{~T}_{1} \mathrm{~T}_{1}\right)$ is rapidly formed within the range of 10 fs to $1 \mathrm{~ns}$ and has a significantly shorter lifetime than that of the free triplet formed via the ISC 
process, although the TA spectrum for the ${ }^{1}\left(\mathrm{~T}_{1} \mathrm{~T}_{1}\right)$ is similar to that of the free triplet formed through ISC. The previous studies for Py in micelles reported the ISC time constant of 1.7 $\mu \mathrm{s},{ }^{41}$ which is much longer than the $\tau_{3}$ time constants of $1.75 \mathrm{~ns}$ and $495.7 \mathrm{ps}$ for the formation of the long-lived species of $A n t i$-DPyB in $n$-hexane and acetonitrile, respectively. Meanwhile, the long-lived species for $S y n$-DPyB in $n$-hexane and acetonitrile formed with $\tau_{3}$ time constants of 9.7 and 8.0 ps, respectively, decay with $\tau_{4}$ time constants of 6.4 and 4.8 ns, respectively. These lifetimes of the long-lived species of Anti-DPyB and Syn-DPyB are significantly shorter than triplet lifetimes of Py $(9.4-11 \mathrm{~ms}) .{ }^{42}$ Considering the faster formation times and shorter lifetimes of the long-lived species of Anti-DPyB compared with Py, we attribute the long-lived species observed from Anti-DPyB and Syn-DPyB to the ${ }^{1}\left(\mathrm{~T}_{1} \mathrm{~T}_{1}\right)$ formed through the first step of SF. Walker et al. showed that SF dynamics of bis(triisopropylsilylethynyl)-tetracene (Tips-tetracene) in solution takes place via an excimer with a ${ }^{1}\left(\mathrm{~T}_{1} \mathrm{~T}_{1}\right)$ character. ${ }^{27}$ Meanwhile, Tips-tetracene in film forms the ${ }^{1}\left(\mathrm{~T}_{1} \mathrm{~T}_{1}\right)$ on ultrafast timescales (in $300 \mathrm{fs}$ ) due to the strong coupling of electronic and vibrational degrees of freedom. ${ }^{28}$ Despite Stern's finding, however, it has been generally accepted that the excimer formation competes with the formation process of ${ }^{1}\left(\mathrm{~T}_{1} \mathrm{~T}_{1}\right),{ }^{23,34}$ indicating that the excimer formation inhibits an SF process. Contrary to this generally accepted view, our results demonstrate that the excimers in both Anti-DPyB and Syn-DPyB are rapidly converted to ${ }^{1}\left(\mathrm{~T}_{1} \mathrm{~T}_{1}\right)$.

\section{Singlet fission.}

The dissociation dynamics of ${ }^{1}\left(\mathrm{~T}_{1} \mathrm{~T}_{1}\right)$ in the SF process is key to determining the energy conversion efficiency in photoelectric or electrochemical devices, as the faster dissociation of ${ }^{1}\left(\mathrm{~T}_{1} \mathrm{~T}_{1}\right)$ to free triplets is preferable in terms of energy conversion efficiency. The nanosecond TA experiments for Anti-DPyB and Syn-DPyB provide a clue for the longlived free triplet generated by the dissociation dynamics of ${ }^{1}\left(\mathrm{~T}_{1} \mathrm{~T}_{1}\right)$. As shown in Supplementary Figures S12 and S14, the absorption bands around $445 \mathrm{~nm}$ of Anti-DPyB are similar to the $\mathrm{T}_{1}$-to- $\mathrm{T}_{\mathrm{n}}$ absorption spectrum of 1-(2-bromophenyl)pyrene measured in DCM. Therefore, we attribute the absorption bands around $445 \mathrm{~nm}$ to the $\mathrm{T}_{1}$-to- $\mathrm{T}_{\mathrm{n}}$ absorption of a free triplet. This result means that free triplets are formed in this system, most likely from the dissociation of ${ }^{1}\left(\mathrm{~T}_{1} \mathrm{~T}_{1}\right)$. In contrast, we could not observe any 
absorption band for $S y n$-DPyB in both $n$-hexane and acetonitrile, suggesting that there are no free triplets at the microsecond time scales. These results indicate that the dissociation dynamics of ${ }^{1}\left(\mathrm{~T}_{1} \mathrm{~T}_{1}\right)$ 's in Syn-DPyB are less favorable than in Anti-DPyB. The suppressed dissociation of ${ }^{1}\left(\mathrm{~T}_{1} \mathrm{~T}_{1}\right)$ into free triplets in $S y n$-DPyB is probably due to the triplet-triplet annihilation, which can be facilitated by the proximity of two Py moieties in the excimer state. Several studies for SF suggested that the triplet-triplet annihilation is one of the decay processes of ${ }^{1}\left(\mathrm{~T}_{1} \mathrm{~T}_{1}\right){ }^{12,43,44,45}$ The triplet-triplet annihilation results in the decay to the ground state and the upconversion to a higher excited singlet state (excimer). The absence of the dissociation of ${ }^{1}\left(\mathrm{~T}_{1} \mathrm{~T}_{1}\right)$ into free triplets in $S y n$-DPyB is probably due to the triplettriplet annihilation. Since the upconversion to the excimer state proceeds with tens picosecond time scale (see the section of Time-resolved TA spectra analysis with kinetic models), we attribute $\tau_{4}$ of Syn-DPyB to the ${ }^{1}\left(\mathrm{~T}_{1} \mathrm{~T}_{1}\right) \rightarrow \mathrm{S}_{0}$ relaxation dynamics.

The difference in SF dynamics of Anti-DPyB and Syn-DPyB may be interpreted in terms of the $E_{\mathrm{b}}$ of ${ }^{1}\left(\mathrm{~T}_{1} \mathrm{~T}_{1}\right)$. According to the kinetic model for SF proposed by Kolomeisly et al., the rates for the formation and dissociation of ${ }^{1}\left(\mathrm{~T}_{1} \mathrm{~T}_{1}\right)$ depend on $E_{\mathrm{b}} .{ }^{46}$ They defined that if $E_{\mathrm{b}}>0$, then the ${ }^{1}\left(\mathrm{~T}_{1} \mathrm{~T}_{1}\right)$ state is bound, and if $E_{\mathrm{b}}<0$, then it is unbound. The ${ }^{1}\left(\mathrm{~T}_{1} \mathrm{~T}_{1}\right)$ destabilization $\left(E_{\mathrm{b}}<0\right)$ makes the fast dissociation of ${ }^{1}\left(\mathrm{~T}_{1} \mathrm{~T}_{1}\right)$ but at the same time makes the slow formation of ${ }^{1}\left(\mathrm{~T}_{1} \mathrm{~T}_{1}\right)$. Based on a spin-lattice model, Abraham and Mayhall predicted that in various CLDs (covalently linked tetracene or pentacene dimers), ${ }^{31}$ the ${ }^{1}\left(\mathrm{~T}_{1} \mathrm{~T}_{1}\right)$ state of meta-linked dimer would be unbound with respect to the separated triplets due to the smaller or negative $E_{\mathrm{b}}$, whereas the ${ }^{1}\left(\mathrm{~T}_{1} \mathrm{~T}_{1}\right)$ states of ortho- and para-linked dimers would be bound. Similarly, the analysis of wave functions by Chesler et al. showed that in CLDs such as para- and meta-bianthracene, the slow formation of ${ }^{1}\left(\mathrm{~T}_{1} \mathrm{~T}_{1}\right)$ in metabianthracene may be due to the small or negative $E_{\mathrm{b}}$, whereas the fast formation of ${ }^{1}\left(\mathrm{~T}_{1} \mathrm{~T}_{1}\right)$ for para-bianthracene results from the larger or positive $E_{\mathrm{b}}{ }^{30}$ In other words, these theoretical studies predicted that compared with ortho- and para-linked dimers, metalinked dimers with a smaller $E_{\mathrm{b}}$ would exhibit a relatively slower formation of ${ }^{1}\left(\mathrm{~T}_{1} \mathrm{~T}_{1}\right)$ state and a relatively fast dissociation of ${ }^{1}\left(\mathrm{~T}_{1} \mathrm{~T}_{1}\right)$ into free triplets with a large yield. Indeed, the experimental results for several CLDs are in agreement with the theoretical predictions. ${ }^{22}$, 30, 31, 32 The theoretical calculation results for many ortho-linked CLDs predict that AntiDPyB and Syn-DPyB, which are also ortho-linked CLDs, will form the bound ${ }^{1}\left(\mathrm{~T}_{1} \mathrm{~T}_{1}\right)$ state 
due to the large or positive $E_{\mathrm{b}}$. Indeed, the experimental results show that the ${ }^{1}\left(\mathrm{~T}_{1} \mathrm{~T}_{1}\right)$ state of $S y n-\mathrm{DPyB}$ is bound. In contrast to $S y n-\mathrm{DPyB}, A n t i-\mathrm{DPyB}$ shows a significantly slower formation of ${ }^{1}\left(\mathrm{~T}_{1} \mathrm{~T}_{1}\right)$ followed by the dissociation into the free triplets, suggesting that the ${ }^{1}\left(\mathrm{~T}_{1} \mathrm{~T}_{1}\right)$ state of Anti-DPyB is unbound. This result indicates that like meta-linked dimers that show favorable SF dynamics, Anti-DPyB, even if it is an ortho-linked dimer, forms the unbound ${ }^{1}\left(\mathrm{~T}_{1} \mathrm{~T}_{1}\right)$ state due to a small $E_{\mathrm{b}}$, leading to the efficient dissociation of ${ }^{1}\left(\mathrm{~T}_{1} \mathrm{~T}_{1}\right)$ into free triplets. The theoretical calculation results by Nakano and coworkers demonstrate that compared with ortho- and para-linked pentacene dimers, the electronic coupling between chromophores for the meta-linked pentacene dimer is very low, resulting in the relatively slow formation of ${ }^{1}\left(\mathrm{~T}_{1} \mathrm{~T}_{1}\right)$ and the efficient $\mathrm{SF} .{ }^{47,}{ }^{48}$ In this regard, the efficient SF in AntiDPyB is due to the low electronic coupling owing to the twisted alignment of two chromophores. This result indicates that the SF dynamics in ortho-linked dimers, which show a significant $\pi$-orbital overlap between two chromophores, can be modulated by controlling the molecular configuration. Consequently, our results for Anti-DPyB and SynDPyB suggest that the molecular geometry of a CLD plays a critical role in their SF dynamics as well as the excimer formation and ICT.

\section{Time-resolved TA spectra analysis with kinetic models.}

To further elucidate the excited-state relaxation dynamics, we analyzed the time-resolved spectra for Anti-DPyB and Syn-DPyB with kinetic models. As discussed in the previous section, the transition from the excimer state to ${ }^{1}\left(\mathrm{~T}_{1} \mathrm{~T}_{1}\right)$ is remarkably faster than the relaxation from the excimer state to the ground state via fluorescence. If the excited-state relaxation dynamics of $A n t i-\mathrm{DPyB}$ and $S y n-\mathrm{DPyB}$ occur with the sequential kinetic model (reaction 1) shown in Scheme 1, the excited molecules in the excimer state may preferentially transit to ${ }^{1}\left(\mathrm{~T}_{1} \mathrm{~T}_{1}\right)$ rather than to the ground state because of the relatively slow relaxation from the excimer state to the ground state. In this case, the excimer emission should not be observed at all. Contrary to this scenario, as shown in Figure 1B, both AntiDPyB and Syn-DPyB exhibit the excimer fluorescences with long lifetimes (Table 1). The significant excimer fluorescences for Anti-DPyB and Syn-DPyB indicate that a sequential kinetic model cannot explain their emission behaviors. As shown in the inset of Figure 2A, furthermore, the rising time of $1.24 \mathrm{~ns}$ in the emission decay profile is approximately five 
times larger than the time constant $(0.23 \mathrm{~ns})$ corresponding to the $\mathrm{S}_{1} \rightarrow$ excimer transition determined from femtosecond TA experiments. This difference indicates that the observed excimer fluorescence is not the prompt emission but the delayed emission. Consequently, we suggest that the dynamics of $1.24 \mathrm{~ns}$ corresponds to the reformation process of the excimer state through a delayed emission channel, suggesting the equilibrium between the excimer state and ${ }^{1}\left(\mathrm{~T}_{1} \mathrm{~T}_{1}\right)$ state (reaction 2 in Scheme 1). On one hand we cannot completely rule out the possibility that the delayed emission is due to the upconversion to the excimer state by the triplet-triplet annihilation. Meanwhile, the absence of the rising features in AntiDPyB in acetonitrile and $S y n$-DPyB in $n$-hexane and acetonitrile implies that the process from ${ }^{1}\left(\mathrm{~T}_{1} \mathrm{~T}_{1}\right)$ state to excimer state is faster than the temporal resolution $(\sim 50 \mathrm{ps})$ of our time-resolved fluorescence measurement system.

To confirm our interpretation, we analyzed the time-resolved TA spectra of Anti-DPyB with a kinetic model (reaction 2 in Scheme 1) containing an equilibrium process between the excimer state and ${ }^{1}\left(\mathrm{~T}_{1} \mathrm{~T}_{1}\right)$ state using the time constants (Table 2) obtained from SVD analysis. Figure 4 shows the species-associated difference spectra (SADS) and population changes for five intermediates (FC, $\mathrm{S}_{1}$, excimer, ${ }^{1}\left(\mathrm{~T}_{1} \mathrm{~T}_{1}\right)$, and $\left.2 \mathrm{~T}_{1}\right)$ for Anti-DPyB in $n$ hexane and acetonitrile. As shown in Supplementary Figure S15, the measured TA spectra for Anti-DPyB are well constructed as a linear combination of the five SADS curves according to the employed kinetic model. These results mean that the excited-state relaxation dynamics of $A n t i$-DPyB in $n$-hexane and acetonitrile are well described with such a kinetic model. In addition, the kinetic analyses demonstrate that in Anti-DPyB, the ${ }^{1}\left(\mathrm{~T}_{1} \mathrm{~T}_{1}\right)$ formed through the excimer state slowly dissociates into free triplets with a $\tau_{4}$ time constant. As shown in Figure 4A, the SADS corresponding to the ${ }^{1}\left(\mathrm{~T}_{1} \mathrm{~T}_{1}\right)$ of Anti-DPyB is different from that corresponding to the free triplet $\left(\mathrm{T}_{1}\right)$, implying that the photophysical properties of the ${ }^{1}\left(\mathrm{~T}_{1} \mathrm{~T}_{1}\right)$ and the free triplet are different.

On the other hand, unlike Anti-DPyB, the ${ }^{1}\left(\mathrm{~T}_{1} \mathrm{~T}_{1}\right)$ in Syn-DpyB does not dissociate into free triplets (see the section of Singlet fission). In addition, the $\tau_{2}$ time constant corresponding to the $\mathrm{S}_{1} \rightarrow$ excimer transition was not observed in Syn-DpyB (Table 2). As mentioned above, the excimer of $S y n$-DPyB with a pre-stacked structure is likely to form fast within a subpicosecond ( $\leq 200 \mathrm{fs}$ ) or with a time constant comparable to IVR ( $3 \mathrm{ps})$. In this regard, we analyzed the time-resolved TA spectra of Syn-DPyB with two kinetic 
models (reactions 3 and 4 in Scheme 1); one is a kinetic model (reaction 3 in Scheme 1) in which the $S_{1} \rightarrow$ excimer transition occurs in subpicosecond ( $\leq 200 \mathrm{fs}$ ), and the other is a kinetic model (reaction 4 in Scheme 1) in which the $S_{1} \rightarrow$ excimer transition occurs with a time constant comparable to IVR $(\sim 3 \mathrm{ps})$. Figure 5 shows the SADSs and population changes for four intermediates (FC, $\mathrm{S} 1$, excimer, and $\left.{ }^{1}\left(\mathrm{~T}_{1} \mathrm{~T}_{1}\right)\right)$ for Syn-DPyB in n-hexane and acetonitrile. The SADSs from the two kinetic models are different, although the measured TA spectra for Syn-DPyB are well explained as a linear combination of the four SADSs of both kinetic models (see Supplementary Figure S16). As shown in Figure 5, the SADS for the $\mathrm{S}_{1}$ state from the kinetic model for reaction 4 is positive, which is consistent with the measured TA spectra, whereas that from the kinetic model for reaction 3 is strongly negative (Supplementary Figure S17), which is contrary to the experimental results. These results from the kinetic analysis suggest that the $\mathrm{S}_{1} \rightarrow$ excimer transition in Syn-DPyB occurs with a time constant comparable to IVR ( 3 ps) and support that the ${ }^{1}\left(\mathrm{~T}_{1} \mathrm{~T}_{1}\right)$ in $S y n$ DPyB returns to the ground state without the dissociation into two free triplets.

\section{Conclusions}

To understand the ultrafast excited-state relaxation dynamics of intramolecular SF materials such as a CLD, we elucidate the ultrafast excited-state relaxation dynamics of covalently linked pyrene dimers, Anti-DPyB and Syn-DPyB. In the excited state, AntiDPyB, in which two Py moieties are oriented in a twisted configuration, forms the excimer through a conformational change with time constants of 231 and 24.3 ps in $n$-hexane and acetonitrile, respectively (Figure 6). Syn-DPyB with a pre-stacked configuration rapidly forms the excimer without any conformational change with a time constant of $\sim 3$ ps. Our results also demonstrate that the excimer emissions observed from Anti-DPyB and SynDPyB are not a prompt emission but a delayed emission. The time-resolved spectroscopic results show that the resulting excimers rapidly relax to the ${ }^{1}\left(\mathrm{~T}_{1} \mathrm{~T}_{1}\right)$ state, suggesting that the ${ }^{1}\left(\mathrm{~T}_{1} \mathrm{~T}_{1}\right) \mathrm{s}$ of $A n t i$-DPyB and Syn-DPyB are formed through the excimer state. The ${ }^{1}\left(\mathrm{~T}_{1} \mathrm{~T}_{1}\right)$ of Anti-DPyB is dissociated to free triplets as the end product, completing SF, whereas SynDPyB does not show the dissociation of ${ }^{1}\left(\mathrm{~T}_{1} \mathrm{~T}_{1}\right)$. This means that Anti-DPyB forms the unbound ${ }^{1}\left(\mathrm{~T}_{1} \mathrm{~T}_{1}\right)$, resulting in the efficient SF dynamics, whereas Syn-DPyB forms the bound ${ }^{1}\left(\mathrm{~T}_{1} \mathrm{~T}_{1}\right)$. The absence of the dissociation of ${ }^{1}\left(\mathrm{~T}_{1} \mathrm{~T}_{1}\right)$ into free triplets in Syn-DPyB is 
probably due to the triplet-triplet annihilation. This result is in contrast to the result predicted from theoretical studies that compared with ortho- and para-linked dimers, metalinked dimers with a smaller $E_{\mathrm{b}}$ exhibit efficient SF dynamics. This finding means that the efficiency of SF dynamics in CLD cannot be predicted only by the substitution position of the chromophore in a CLD. Our results show that compared with Syn-DpyB, the efficient SF in Anti-DPyB is due to the relatively low electronic coupling owing to the twisted alignment of two chromophores. This result indicates that the SF dynamics in ortho-linked dimers, which generally shows a significant $\pi$-orbital overlap between two chromophores, can be modulated by the control of the molecular configuration, consequently suggesting that the molecular geometry of a CLD plays a critical role in their SF dynamics as well as the excimer formation, and ICT.

\section{Author Contributions}

J.C., K.-R.W., and H.I. designed research; J.C., S.K., M.A., J.K., D.W.C., K.-R.W., and H.I. performed research; J.C., S.K., M.A., J.K., D.W.C., K.-R.W., and H.I. contributed interpretation of results and J.C., K.-R.W., and H.I. wrote the paper.

\section{Conflicts of interest}

There are no conflicts to declare.

\section{Acknowledgements}

This work was supported by the Basic Science Research Program through the National Research Foundation of Korea (NRF), funded by the Ministry of Education (NRF2017R1C1B1010736, NRF-2020R1C1C1009007 and NRF-2020R1I1A3054352). This work was supported by the Institute for Basic Science (IBS-R004).

\section{Notes}

CCDC 2089494 contains the supplementary crystallographic data for this paper. These data can be obtained free of charge from The Cambridge Crystallographic Data Centre via www.ccdc.cam.ac.uk/data_request/cif. 


\section{References}

1. $\mathrm{Yu} \mathrm{Y,} \mathrm{et} \mathrm{al.} \mathrm{Fast} \mathrm{Photoelectric} \mathrm{Conversion} \mathrm{in} \mathrm{the} \mathrm{Near-Infrared} \mathrm{Enabled} \mathrm{by} \mathrm{Plasmon-}$ Induced Hot-Electron Transfer. Adv. Mater. 31, 1903829 (2019).

2. Gasparini N, et al. Designing Ternary Blend Bulk Heterojunction Solar Cells with Reduced Carrier Recombination and a Fill Factor of 77\%. Nat. Energy 1, (2016).

3. Li YR, et al. Implementing Metal-to-Ligand Charge Transfer in Organic Semiconductor for Improved Visible-Near-Infrared Photocatalysis. Adv. Mater. 28, 6959-6965 (2016).

4. Ni ZY, et al. Plasmonic Silicon Quantum Dots Enabled High-Sensitivity Ultrabroadband Photodetection of Graphene-Based Hybrid Phototransistors. ACS Nano 11, 9854-9862 (2017).

5. Cheng RQ, et al. High-Performance, Multifunctional Devices Based on Asymmetric van der Waals Heterostructures. Nat. Electron. 1, 356-361 (2018).

6. Ren P, Sun C, Shi Y, Song P, Yang Y, Li Y. Global Performance Evaluation of Solar Cells Using Two Models: from Charge-Transfer and Recombination Mechanisms to Photoelectric Properties. J. Mater. Chem. C 7, 1934-1947 (2019).

7. Rao A, Friend RH. Harnessing Singlet Exciton Fission to Break the Shockley-Queisser Limit. Nat. Rev. Mater. 2, (2017).

8. Grabowski ZR, Rotkiewicz K, Rettig W. Structural Changes Accompanying Intramolecular Electron Transfer: Focus on Twisted Intramolecular Charge-Transfer States and Structures. Chem. Rev. 103, 3899-4031 (2003).

9. Kim S, Ahn D-S, Ahn M, Wee K-R, Choi J, Ihee H. Charge Transfer Induced by Electronic State Mixing in a Symmetric X-Y-X-type Multi-chromophore System. Phys. Chem. Chem. Phys. 22, 28440-28447 (2020).

10. Adams DM, et al. Charge Transfer on the Nanoscale: Current Status. J. Phys. Chem. B 107, 6668-6697 (2003).

11. Brédas J-L, Beljonne D, Coropceanu V, Cornil J. Charge-Transfer and Energy-Transfer Processes in $\pi$-Conjugated Oligomers and Polymers: A Molecular Picture. Chem. Rev. 104, 4971-5004 (2004).

12. Monahan N, Zhu XY. Charge Transfer-Mediated Singlet Fission. Annu. Rev. Phys. Chem. 66, 601-618 (2015).

13. Holten D, Bocian DF, Lindsey JS. Probing Electronic Communication in Covalently Linked Multiporphyrin Arrays. A Guide to the Rational Design of Molecular Photonic Devices. Acc. Chem. Res. 35, 57-69 (2002). 
14. Popp W, Brey D, Binder R, Burghardt I. Quantum Dynamics of Exciton Transport and Dissociation in Multichromophoric Systems. Annu. Rev. Phys. Chem. 72, 591-616 (2021).

15. Oh I, et al. Enhancement of Energy Transfer Efficiency with Structural Control of Multichromophore Light-Harvesting Assembly. Adv. Sci. 7, 2001623 (2020).

16. Young RM, Wasielewski MR. Mixed Electronic States in Molecular Dimers: Connecting Singlet Fission, Excimer Formation, and Symmetry-Breaking Charge Transfer. Acc. Chem. Res. 53, 1957-1968 (2020).

17. Kim S-T, Hartman RF, Rose SD. Solvent Dependence of Pyrimidine Dimer Splitting in a Covalently Linked Dimer-Indole System. Photochem. Photobiol. 52, 789-794 (1990).

18. Kumarasamy E, et al. Tuning Singlet Fission in $\pi$-Bridge- $\pi$ Chromophores. J. Am. Chem. Soc. 139, 12488-12494 (2017).

19. Hong Y, et al. Efficient Multiexciton State Generation in Charge-Transfer-Coupled Perylene Bisimide Dimers via Structural Control. J. Am. Chem. Soc. 142, 7845-7857 (2020).

20. Margulies EA, et al. Direct Observation of a Charge-Transfer State Preceding High-Yield Singlet Fission in Terrylenediimide Thin Films. J. Am. Chem. Soc. 139, 663-671 (2017).

21. Smith MB, Michl J. Recent Advances in Singlet Fission. Annu. Rev. Phys. Chem. 64, 361386 (2013).

22. Zirzlmeier J, et al. Singlet Fission in Pentacene Dimers. Proc. Natl. Acad. Sci. U.S.A. 112, 5325-5330 (2015).

23. Margulies EA, et al. Enabling Singlet Fission by Controlling Intramolecular Charge Transfer in $\pi$-Stacked Covalent Terrylenediimide Dimers. Nat. Chem. 8, 1120-1125 (2016).

24. Busby E, et al. A Design Strategy for Intramolecular Singlet Fission Mediated by ChargeTransfer States in Donor-Acceptor Organic Materials. Nat. Mater. 14, 426-433 (2015).

25. Smith MB, Michl J. Singlet Fission. Chem. Rev. 110, 6891-6936 (2010).

26. Johnson JC, Nozik AJ, Michl J. The Role of Chromophore Coupling in Singlet Fission. Acc. Chem. Res. 46, 1290-1299 (2013).

27. Walker BJ, Musser AJ, Beljonne D, Friend RH. Singlet Exciton Fission in Solution. Nat. Chem. 5, 1019-1024 (2013).

28. Stern HL, et al. Vibronically Coherent Ultrafast Triplet-Pair Formation and Subsequent Thermally Activated Dissociation Control Efficient Endothermic Singlet Fission. Nat. Chem. 9, 1205-1212 (2017). 
29. Schultz JD, et al. Influence of Vibronic Coupling on Ultrafast Singlet Fission in a Linear Terrylenediimide Dimer. J. Am. Chem. Soc. 143, 2049-2058 (2021).

30. Chesler R, Khan S, Mazumdar S. Wave Function Based Analysis of Dynamics versus Yield of Free Triplets in Intramolecular Singlet Fission. J. Phys. Chem. A 124, 1009110099 (2020).

31. Abraham V, Mayhall NJ. Simple Rule To Predict Boundedness of Multiexciton States in Covalently Linked Singlet-Fission Dimers. J. Phys. Chem. Lett. 8, 5472-5478 (2017).

32. Zirzlmeier J, et al. Solution-based Intramolecular Singlet Fission in Cross-Conjugated Pentacene Dimers. Nanoscale 8, 10113-10123 (2016).

33. Korovina NV, et al. Linker-Dependent Singlet Fission in Tetracene Dimers. J. Am. Chem. Soc. 140, 10179-10190 (2018).

34. Ni W, Gurzadyan GG, Zhao J, Che Y, Li X, Sun L. Singlet Fission from Upper Excited Electronic States of Cofacial Perylene Dimer. J. Phys. Chem. Lett. 10, 2428-2433 (2019).

35. Jo S, Ahn M, Bhattarai K, Wee KR, Ahn DH, Song JW. UV/Vis Absorption Spectrum Calculations of Benzo-1,2-dipyrene Isomer Uusing Long-range Corrected Density Functional Theory. Chem. Phys. Lett. 761, (2020).

36. Crawford AG, et al. Experimental and Theoretical Studies of the Photophysical Properties of 2- and 2,7-Functionalized Pyrene Derivatives. J. Am. Chem. Soc. 133, 13349-13362 (2011).

37. Birks JB, Christophorou LG. Excimer Fluorescence Spectra of Pyrene Derivatives. Spectrochimica Acta 19, 401-410 (1963).

38. Van Dyke DA, Pryor BA, Smith PG, Topp MR. Nanosecond Time-Resolved Fluorescence Spectroscopy in the Physical Chemistry Laboratory: Formation of the Pyrene Excimer in Solution. J. Chem Educ. 75, 615 (1998).

39. D'Aleo A, Cecchetto E, De Cola L, Williams RM. Metal Ion Enhanced Charge Transfer in a Terpyridine-bis-Pyrene System. Sensors 9, 3604-3626 (2009).

40. Rajagopal SK, Mallia AR, Hariharan M. Enhanced Intersystem Crossing in Carbonylpyrenes. Phys. Chem. Chem. Phys. 19, 28225-28231 (2017).

41. Nakamura T, Kira A, Imamura M. Enhancement of the Intersystem Crossing of Pyrene by Metal Ions in Sodium Dodecyl Sulfate Micelle Solutions. J. Phys. Chem. 86, 3359-3363 (1982). 
42. Langelaar J, Rettschnick RPH, Hoijtink GJ. Studies on Triplet Radiative Lifetimes, Phosphorescence, and Delayed Fluorescence Yields of Aromatic Hydrocarbons in Liquid Solutions. J. Chem. Phys. 54, 1-7 (1971).

43. Tamura H. Triplet Exciton Transfers and Triplet-Triplet Annihilation in Anthracene Derivatives via Direct versus Superexchange Pathways Governed by Molecular Packing. J. Phys. Chem. A 124, 7943-7949 (2020).

44. Lin H-H, Kue KY, Claudio GC, Hsu C-P. First Principle Prediction of Intramolecular Singlet Fission and Triplet Triplet Annihilation Rates. J. Chem Theory Comput. 15, 22462253 (2019).

45. Müller AM, Avlasevich YS, Schoeller WW, Müllen K, Bardeen CJ. Exciton Fission and Fusion in Bis(tetracene) Molecules with Different Covalent Linker Structures. J. Am. Chem. Soc. 129, 14240-14250 (2007).

46. Kolomeisky AB, Feng X, Krylov AI. A Simple Kinetic Model for Singlet Fission: A Role of Electronic and Entropic Contributions to Macroscopic Rates. J. Phys. Chem. C 118, 5188-5195 (2014).

47. Ito S, Nagami T, Nakano M. Design Principles of Electronic Couplings for Intramolecular Singlet Fission in Covalently-Linked Systems. J. Phys. Chem. A 120, 6236-6241 (2016).

48. Ito S, Nagami T, Nakano M. Rational Design of Doubly-Bridged Chromophores for Singlet Fission and Triplet-Triplet Annihilation. RSC. Adv. 7, 34830-34845 (2017). 


\section{Table captions}

Table 1. Emission quantum yields $(\Phi)$, emission lifetimes $(\tau)$, average emission lifetimes $(\langle\tau\rangle)$, radiative rate constants $\left(k_{\mathrm{R}}\right)$, and nonradiative rate constants $\left(k_{\mathrm{NR}}\right)$ of Anti-DpyB and Syn-DPyB in $n$-hexane and acetonitrile

Table 2. Time constants determined from TA measurements for Anti-DPyB and Syn-DPyB in $n$ hexane and acetonitrile 


\section{Scheme caption}

Scheme 1. Photo-induced reaction schemes for Anti-DPyB and Syn-DPyB. ( $\mathrm{S}_{0}$ : ground state, FC: Franck-Condon state, $S_{1}$ : singlet excited state, ${ }^{1}\left(\mathrm{~T}_{1} \mathrm{~T}_{1}\right)$ : correlated triplet pair, and $\mathrm{T}_{1}$ : free triplet state). 


\section{Figures captions}

Figure 1. Structures and UV-visible absorption (solid line) and emission spectra (dotted line). (A) Geometric isomers of anti-1,2-di(phenyl) benzene (Anti-DPyB) and syn-1,2-di(phenyl) benzene (Syn-DPyB). (B) UV-visible absorption spectra of Anti- DPyB and Syn-DPyB in $n$-hexane (black) and acetonitrile (red). Emission spectra of Anti-DPyB and Syn-DPyB in $n$-hexane (black) and acetonitrile (red) $\left(\lambda_{\mathrm{ex}}=345 \mathrm{~nm}\right)$.

Figure 2. Fluorescence decay profiles. (A) Fluorescence decay profiles of Anti-DPyB in $n$-hexane (black) and acetonitrile (blue), respectively. In Figure 2A, the inset shows the fluorescence decay profile of Anti-DPyB measured at the long-wavelength $(500 \sim 600 \mathrm{~nm})$ in $n$-hexane. The fluorescence decay profile of Anti-DPyB measured at the long-wavelength $(500 \sim 600 \mathrm{~nm})$ shows a rise time of $1.24 \mathrm{~ns}$ as well as the decay time of $11.1 \mathrm{~ns}$. (B) Fluorescence decay profiles of SynDPyB in $n$-hexane (black) and acetonitrile (blue), respectively.

Figure 3. Transient absorption spectra. (A, B) Transient absorption spectra of Anti-DPyB in $n$ hexane and acetonitrile, respectively. (C, D) Transient absorption spectra of Syn-DPyB in $n$-hexane and acetonitrile, respectively.

Figure 4. Species-associated difference spectra and population changes of intermediates for AntiDPyB in $n$-hexane and acetonitrile. (A, B) Species-associated difference spectra in (A) $n$-hexane and (B) acetonitrile. (C, D) Population changes of intermediates in (C) $n$-hexane and (D) acetonitrile. The solid lines are the concentrations obtained from the kinetics analysis. The open circles represent the measure time delays.

Figure 5. Species-associated difference spectra and population changes of intermediates for SynDPyB in $n$-hexane and acetonitrile. (A, B) Species-associated difference spectra in (A) $n$-hexane and (B) acetonitrile. (C, D) Population changes of intermediates in (C) $n$-hexane and (D) acetonitrile. The solid lines are the concentrations obtained from the kinetics analysis. The open circles represent the measure time delays.

Figure 6. Proposed schematic relaxation dynamics of Anti-DPyB and Syn-DPyB. Excited-state relaxation dynamics of (A) Anti-DPyB and (B) Syn-DPyB. IVR stands for intramolecular vibrational relaxation. 
Table 1. Emission quantum yields $(\Phi)$, emission lifetimes $(\tau)$, average emission lifetimes $(\langle\tau\rangle)$, radiative rate constants $\left(k_{\mathrm{R}}\right)$, and nonradiative rate constants $\left(k_{\mathrm{NR}}\right)$ of Anti-DpyB and Syn-DPyB in $n$-hexane and acetonitrile

\begin{tabular}{c|c|c|c|c|c|c|c}
\hline & \multirow{2}{*}{$\boldsymbol{\Phi}$} & \multicolumn{3}{|c|}{$\tau(\mathbf{n s})$} & $\begin{array}{c}\boldsymbol{k}_{\mathbf{R}}+ \\
\left(\times \mathbf{1 0}^{7} \mathbf{s}^{-1}\right)\end{array}$ & $\begin{array}{c}\boldsymbol{k}_{\mathbf{N R}} \neq \\
\left(\times \mathbf{1 0}^{7} \mathbf{s}^{-1}\right)\end{array}$ \\
\cline { 3 - 8 } Anti-DPyB & in $n$-hexane & 0.20 & $1.68 \pm 0.03$ & $11.12 \pm 0.11$ & 4.23 & 4.73 & 18.9 \\
\hline & in acetonitrile & 0.21 & $0.74 \pm 0.02$ & $12.50 \pm 0.07$ & 3.33 & 6.31 & 23.7 \\
\hline \multirow{2}{*}{$\boldsymbol{S y n}$-DPyB } & in $n$-hexane & 0.09 & $1.89 \pm 0.04$ & $7.76 \pm 0.10$ & 3.59 & 2.51 & 25.3 \\
\hline & in acetonitrile & 0.14 & $2.93 \pm 0.10$ & $8.64 \pm 0.21$ & 4.87 & 2.87 & 17.7 \\
\hline
\end{tabular}

$+k_{R}=\Phi /\langle\tau\rangle$

$\neq k_{\mathrm{NR}}=(1-\Phi) /\langle\tau\rangle$ 
Table 2. Time constants determined from TA measurements for Anti-DPyB and Syn-DPyB in $n$ hexane and acetonitrile

\begin{tabular}{|c|c|c|c|c|c|}
\hline & & $\tau_{1} / \mathrm{ps}$ & $\tau_{2} / \mathrm{ps}$ & $\tau_{3} / \mathrm{ps}$ & $\tau_{4} / \mathrm{ns}$ \\
\hline \multirow{2}{*}{ Anti-DPyB } & in $n$-hexane & $3.6 \pm 0.3$ & $231 \pm 19$ & $1750 \pm 116$ & $>10$ \\
\hline & in acetonitrile & $2.8 \pm 0.1$ & $24.3 \pm 0.5$ & $495.7 \pm 6.5$ & $>10$ \\
\hline \multirow{2}{*}{ Syn-DPyB } & in $n$-hexane & $2.3 \pm 0.8$ & - & $9.7 \pm 0.5$ & $6.4 \pm 0.2$ \\
\hline & in acetonitrile & 2.8 & - & $8.0 \pm 0.6$ & $4.8 \pm 0.2$ \\
\hline
\end{tabular}




\section{In Anti-DPyB:}

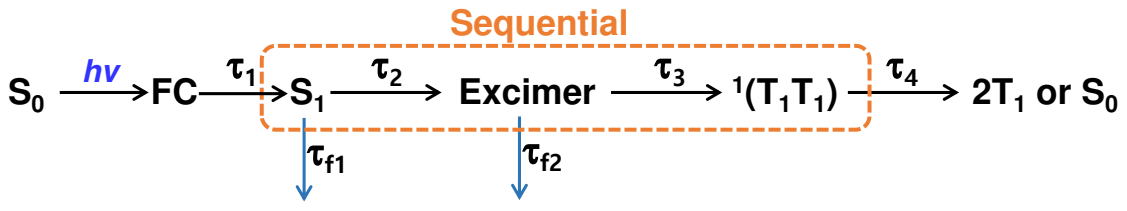

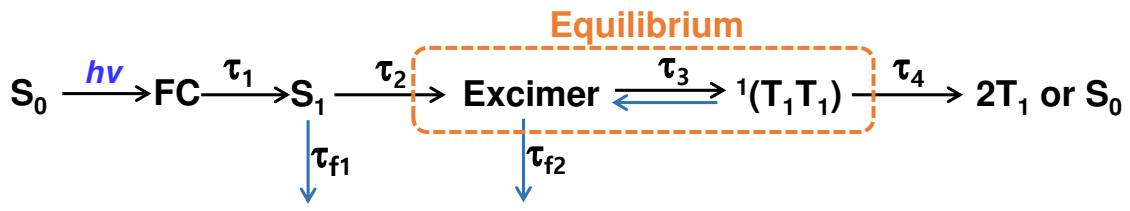

In Syn-DPyB:

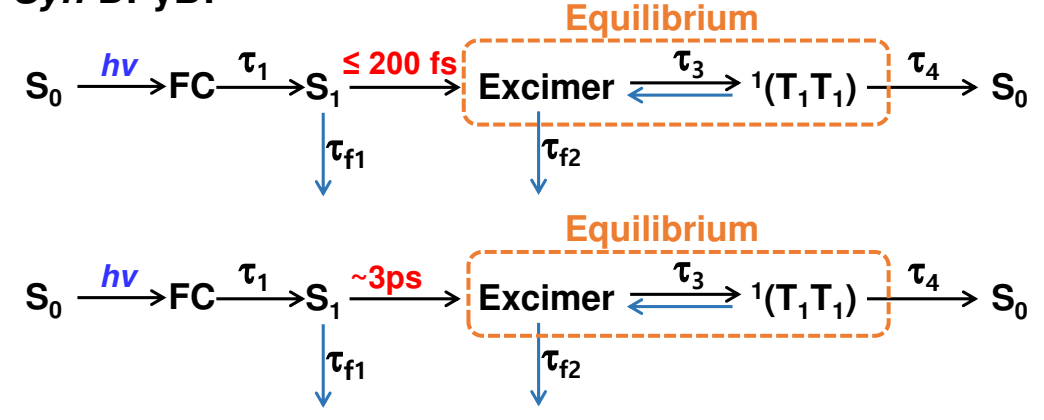

Scheme 1. Photo-induced reaction schemes for Anti-DPyB and Syn-DPyB. ( $\mathrm{S}_{0}$ : ground state, FC: Franck-Condon state, $\mathrm{S}_{1}$ : singlet excited state, ${ }^{1}\left(\mathrm{~T}_{1} \mathrm{~T}_{1}\right)$ : correlated triplet pair, and $\mathrm{T}_{1}$ : free triplet state). 


\section{Figures}

A)

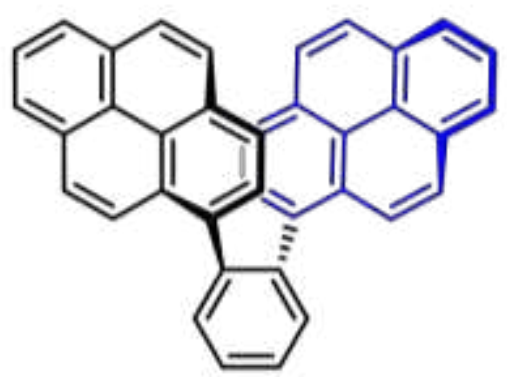

anti-1,2-di(pyrenyl)benzene (Anti-DPyB)

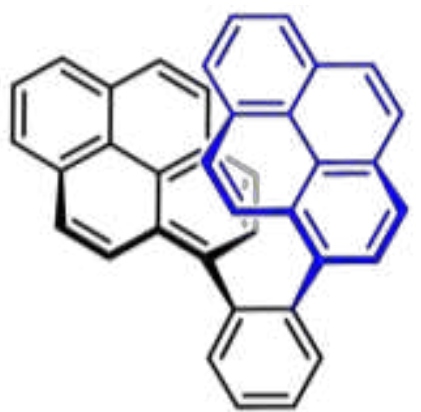

syn-1,2-di(pyrenyl)benzene (Syn-DPyB)

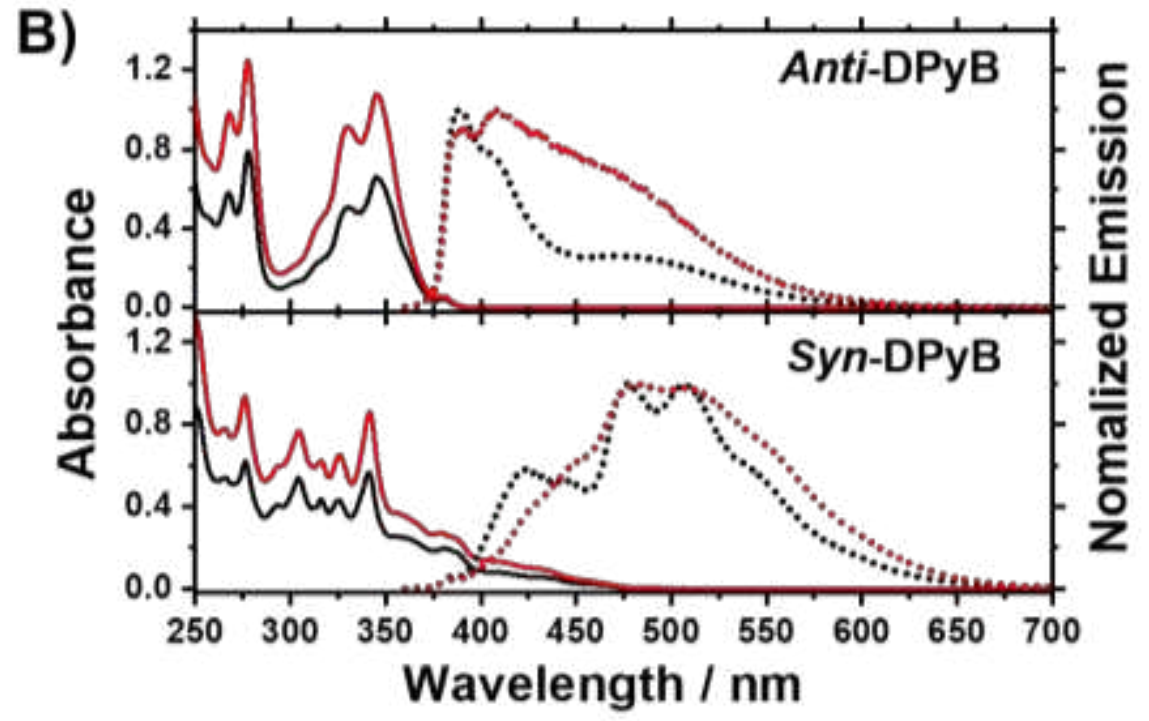

Figure 1. Structures and UV-visible absorption (solid line) and emission spectra (dotted line). (A) Geometric isomers of anti-1,2-di(phenyl) benzene (Anti-DPyB) and syn-1,2-di(phenyl) benzene (Syn-DPyB). (B) UV-visible absorption spectra of Anti- DPyB and Syn-DPyB in $n$-hexane (black) and acetonitrile (red). Emission spectra of Anti-DPyB and Syn-DPyB in $n$-hexane (black) and acetonitrile (red) $\left(\lambda_{\mathrm{ex}}=345 \mathrm{~nm}\right)$. 

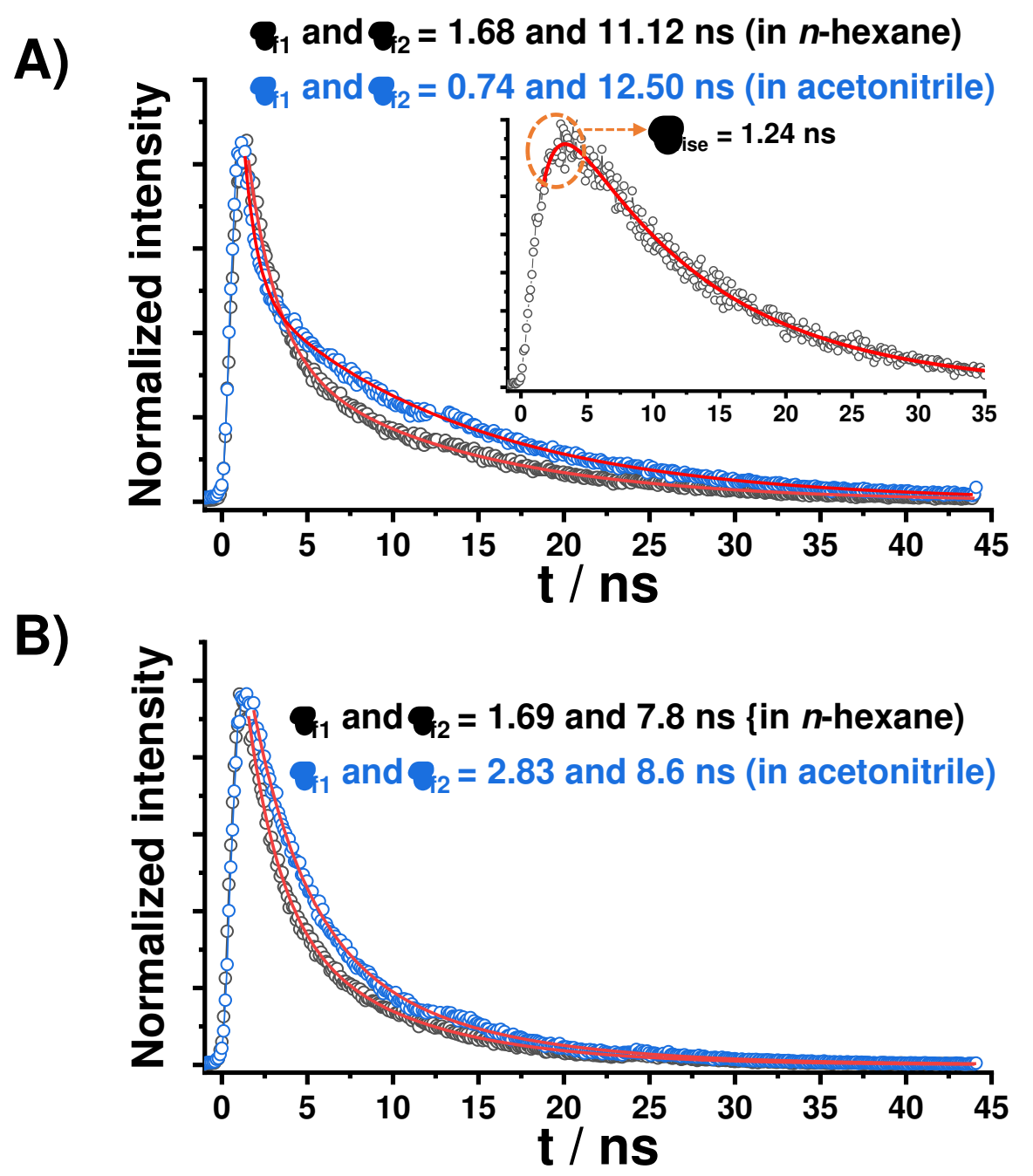

Figure 2. Fluorescence decay profiles. (A) Fluorescence decay profiles of Anti-DPyB in $n$-hexane (black) and acetonitrile (blue), respectively. In Figure 2A, the inset shows the fluorescence decay profile of Anti-DPyB measured at the long-wavelength $(500 \sim 600 \mathrm{~nm})$ in $n$-hexane. The fluorescence decay profile of Anti-DPyB measured at the long-wavelength $(500 \sim 600 \mathrm{~nm})$ shows a rise time of $1.24 \mathrm{~ns}$ as well as the decay time of $11.1 \mathrm{~ns}$. (B) Fluorescence decay profiles of SynDPyB in $n$-hexane (black) and acetonitrile (blue), respectively. 

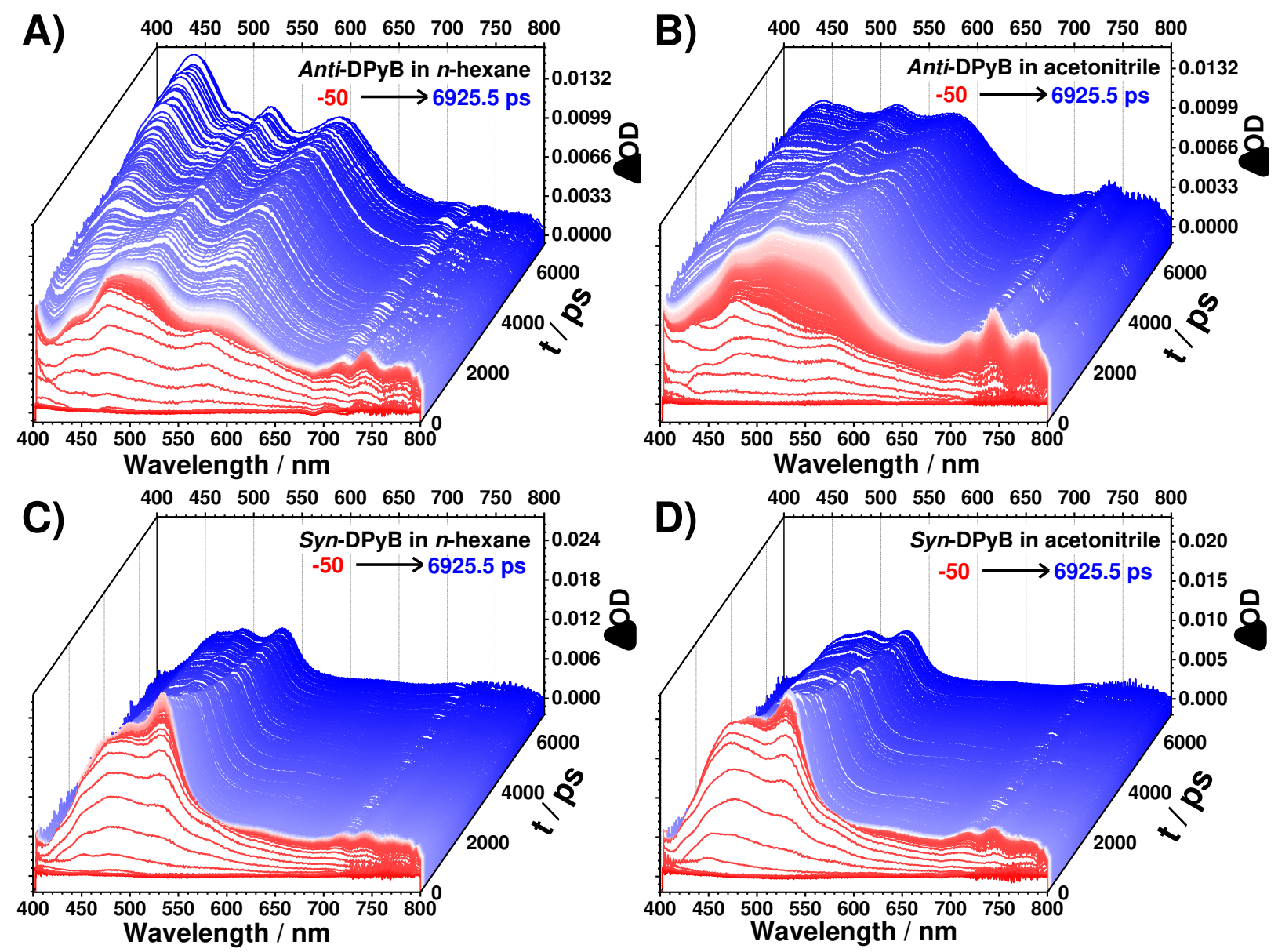

Figure 3. Transient absorption spectra. (A, B) Transient absorption spectra of Anti-DPyB in $n$ hexane and acetonitrile, respectively. (C, D) Transient absorption spectra of $S y n$-DPyB in $n$-hexane and acetonitrile, respectively. 

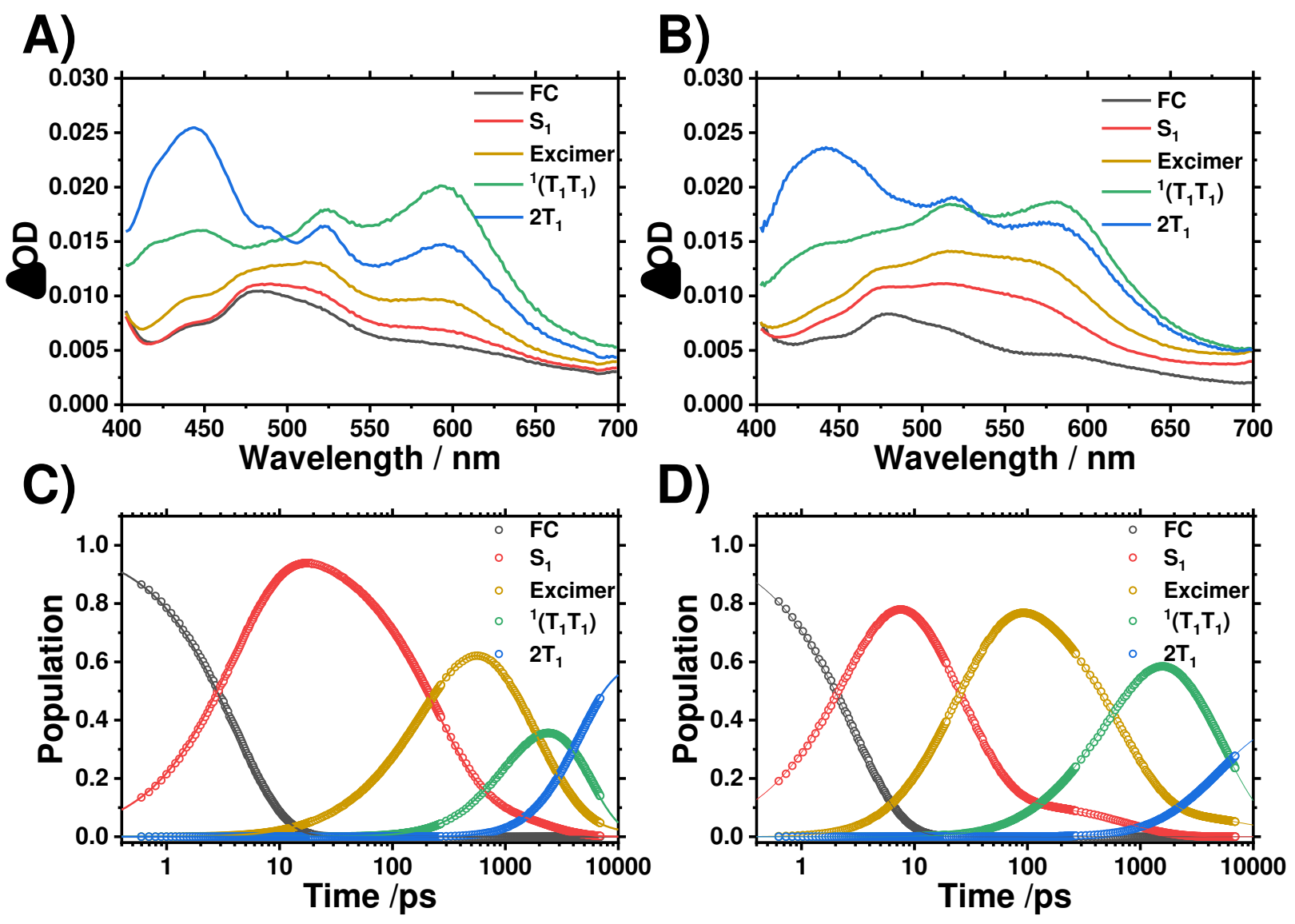

Figure 4. Species-associated difference spectra and population changes of intermediates for AntiDPyB in $n$-hexane and acetonitrile. (A, B) Species-associated difference spectra in (A) $n$-hexane and (B) acetonitrile. (C, D) Population changes of intermediates in (C) $n$-hexane and (D) acetonitrile. The solid lines are the concentrations obtained from the kinetics analysis. The open circles represent the measure time delays. 

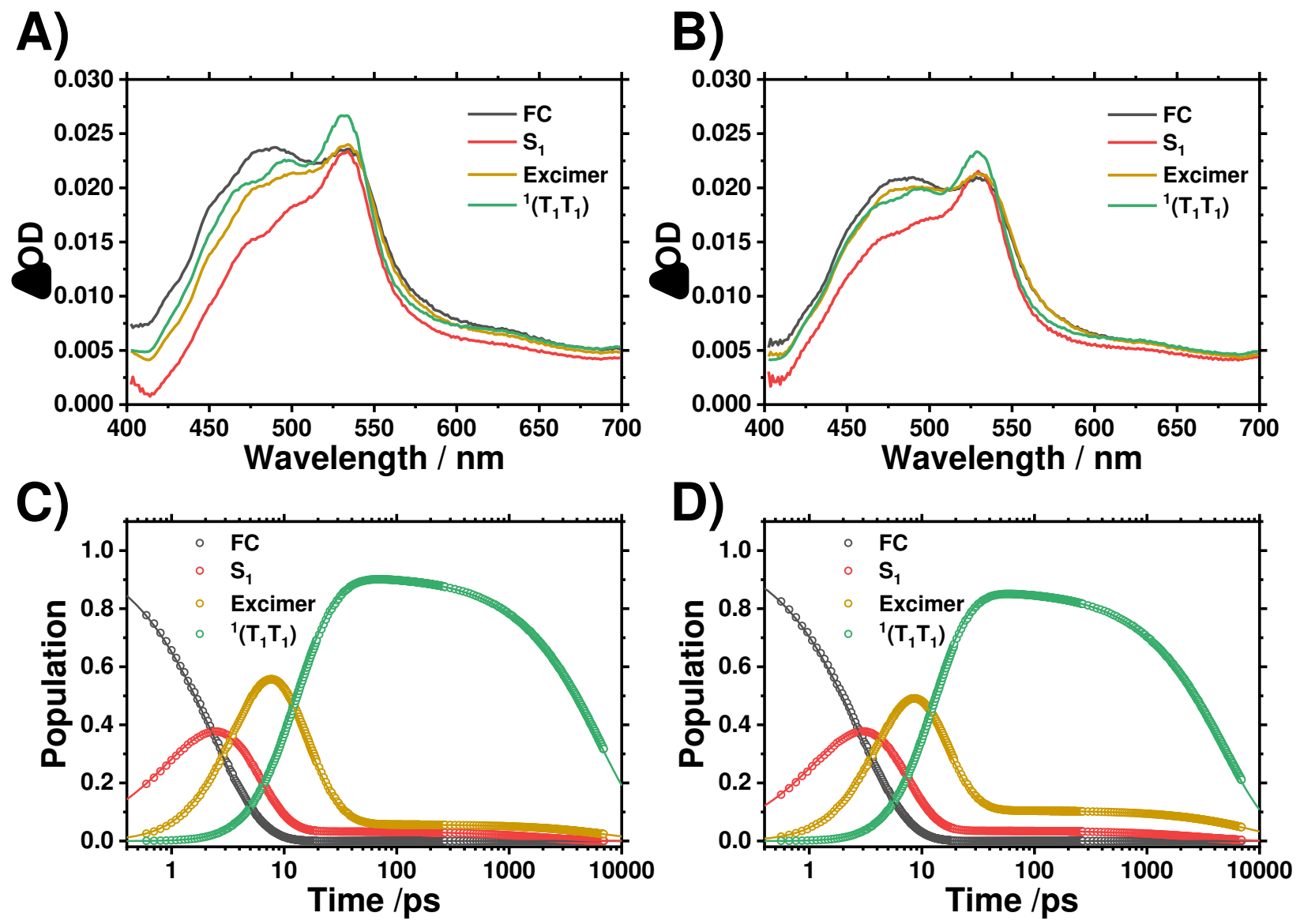

Figure 5. Species-associated difference spectra and population changes of intermediates for SynDPyB in $n$-hexane and acetonitrile. (A, B) Species-associated difference spectra in (A) $n$-hexane and (B) acetonitrile. (C, D) Population changes of intermediates in (C) $n$-hexane and (D) acetonitrile. The solid lines are the concentrations obtained from the kinetics analysis. The open circles represent the measure time delays. 

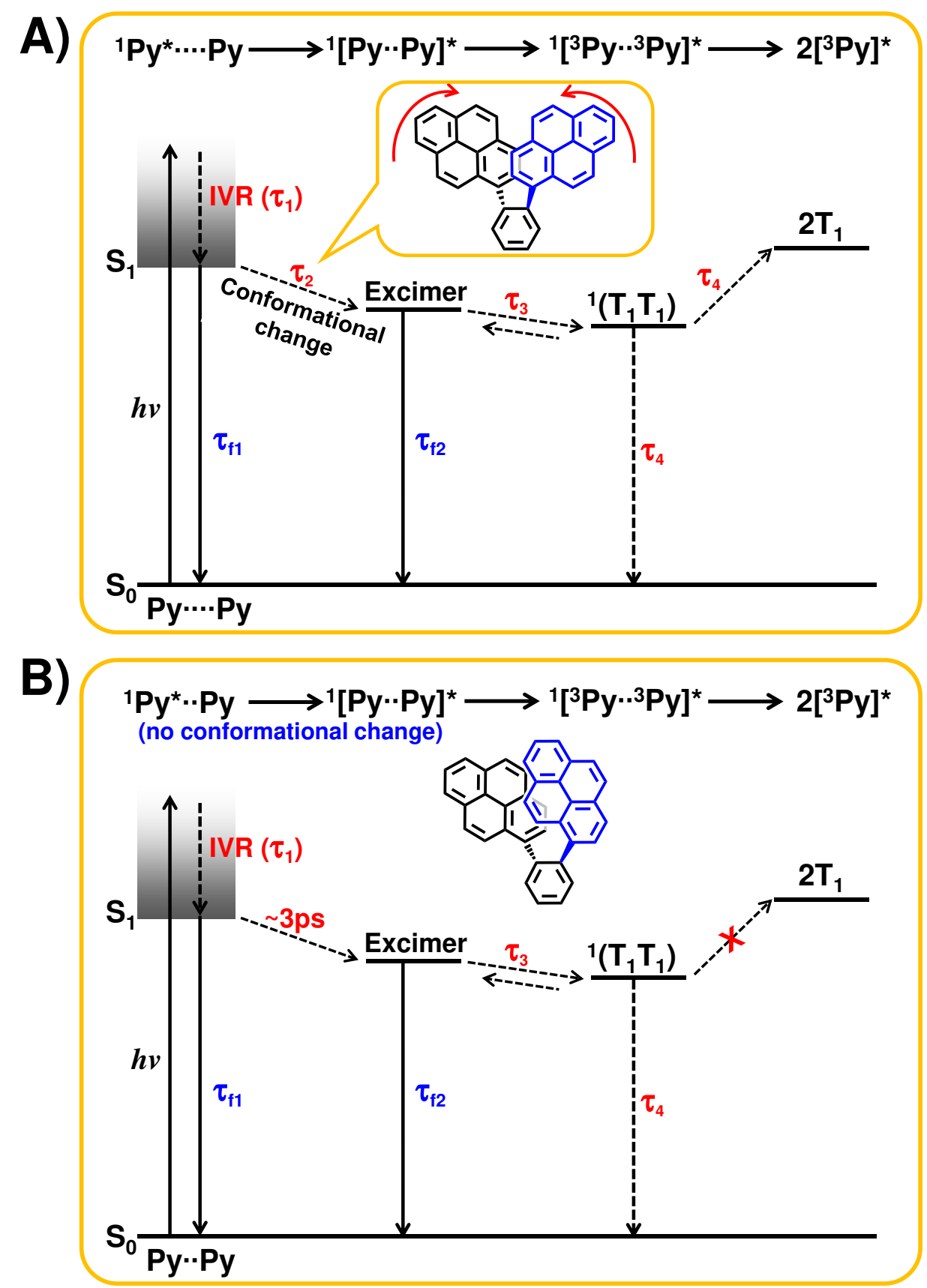

Figure 6. Proposed schematic relaxation dynamics of Anti-DPyB and Syn-DPyB. Excited-state relaxation dynamics of (A) Anti-DPyB and (B) Syn-DPyB. IVR stands for intramolecular vibrational relaxation. 


\section{Supplementary Files}

This is a list of supplementary files associated with this preprint. Click to download.

- SFDPyBCommunChemSI.docx 